\title{
A comprehensive kinetic model for ternary complex catalysis
}

\author{
Eugene F. Douglass Jr.; ${ }^{1, *}$ Chad J. Miller ${ }^{2}$
}

${ }^{1}$ Pharmaceutical and Biomedical Sciences, University of Georgia, 250 W. Green Street, Athens, GA 30602, USA.

${ }^{2}$ Department of Biochemistry \& Institute for Protein Design, University of Washington, Seattle, WA 98195, USA.

*corresponding author (eugene.douglass@uga.edu) 


\section{Abstract}

Ternary-complex directed enzyme Integrated Michaelis Menten $\begin{aligned} & \text { SPED } \\ & \text { LMIT }\end{aligned} \quad \oint_{\text {efficiency }}^{\text {targeting }}$ catalysis underlies a vast array of biological processes and several clinical therapies including growth hormones, interferon, and heparin. Recently, interest in ternarycatalysis drugs has increased significantly with the rapid expansion of research new technologies such as bispecific antibodies and proteolysis targeting chimeras (PROTAC's).

Here, we derive a general model for ternary-

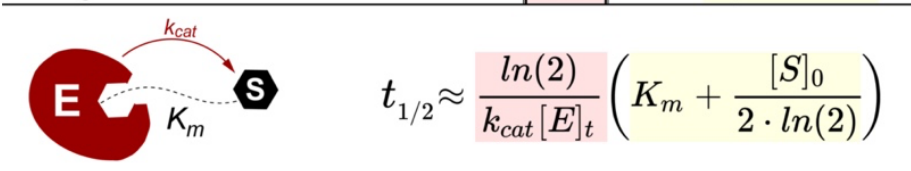

Integrated Ternary Kinetics (Presented Here)

complex catalysis that defines the timescales of these diverse processes in familiar terms from classical enzyme theory. This was accomplished by solving for the maximum velocity $\left(V_{\max }\right)$ and adapting an underappreciated strategy within Michaels and Menten's original publication: integration of the velocity equation. Critically, these equations are simple, conceptually accessible, and enables rapid estimation timescales that are consistent with a wide range of published literature. Finally, we have combined these equations with "big data" from new thermodynamic and kinetic databases to build interactive online tools that enable non-computational investigators to graphically simulate their own systems:

- https://douglasslab.com/Btmax kinetics/

Overall, this work is part of a general trend to reconceptualize pharmacodynamics from classical bindingequilibria (e.g. Langmuir-Hill equation) to a kinetic processes with a characteristic timescale. 
Ternary-complex directed Enzyme Catalysis (TEC) underlies a wide range of biological and clinical processes. TEC is mediated by a bifunctional molecule (B) that simultaneously binds and an enzyme or effector $(E)$ and a target $(T)$, forming a ternary complex that directs enzymatic modification of the target (Figure 1). This mechanism underlies inter-cellular signaling of most cytokines and hormones and is ubiquitous in the intra-cellular signaling of MAP-kinase cascades, calcium signaling and several stress-response pathways. ${ }^{1,2}$ In addition to natural processes, several clinical drugs employ TEC including several biologicals such as heparin, interferon, growth hormones and monoclonal antibodies. ${ }^{3-}$ ${ }^{8}$ Finally, the efficacy of several natural and synthetic small molecules has been shown to be dependent on the formation of a ternary complex including: rapamycin, cyclosporine and lenalidomide. ${ }^{9}$

Recently, interest in developing synthetic ternary complex-based drugs has increased significantly., ${ }^{9,10}$ Prominent examples include both biological and chemical agents such as bispecific antibodies (bsAbs) and PROteolysis TArgeting Chimera's (PROTACs), respectively. ${ }^{6,11}$ BsAbs can simultaneously bind the CD3 receptor of T-cells and tumor antigens on cancer cells; this colocalization directs anticancer immune responses in the absence of classical T-cell activation or TCR specificity. PROTACs simultaneously bind E3 ubiquitin ligases and oncogenic proteins, causing ubiquitination and degradation of target proteins. BsAbs first entered the clinic in 2009, and now over 40 different bsAb's are currently in clinical development. ${ }^{6,12}$ In 2019, PROTACs began initial clinical validation, and it has been estimated that 15 new PROTAC-based clinical trials will begin by the end of $2021 .^{13}$ Overall, this new clinical

A.

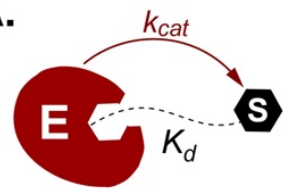

C.

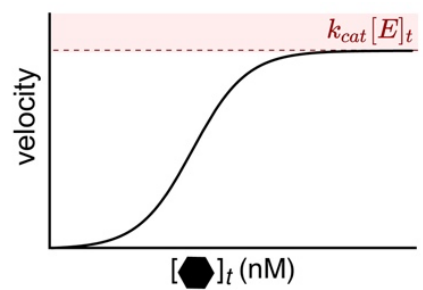

B.

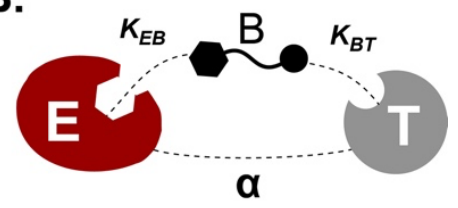

D.

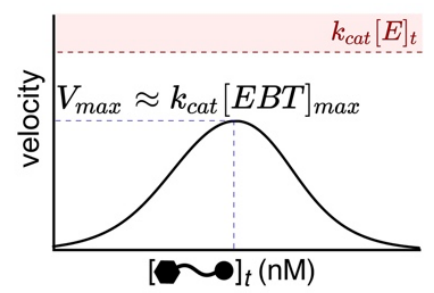

Figure 1. The kinetics of binary and ternary systems are distinct. A. Classical Enzyme kinetics assumes that one enzyme directly binds to and acts on one target B. Scaffolded enzyme kinetics involved a third molecule (B) that indirectly links the enzyme $(E)$ and its downstream target $(\mathrm{T}) \mathrm{C}$. In binary systems, increasing the substrate concentration increases the rate of the reaction, up to $V$ max, which equals $k_{c a t}[E]_{t}$ in classical Michaelis-Menten kinetics $\mathbf{D}$. In ternary SEC, the reaction rate follows a bell-shaped doseresponse curves.

focus on synthetic ternary-complex mediated catalysis has renewed interest in the kinetic models that underly TEC.

\section{Classical Models of Enzyme Kinetics}

Michaelis and Menten first quantitatively described enzyme catalysis in 1913 (Figure 1A). ${ }^{14,15}$ Classical kinetic models assume that the enzyme $(E)$ binds directly to its substrate $(S)$ with a binding affinity $K_{d}$ (Figure 1A). This binary complex mechanism is responsible for the familiar "saturation doseresponse curves" where excess substrates bind all available enzyme (Figure 1C). Critically, the Michaelis-Menten model was derived by assuming that the initial substrate concentration was in excess of the total enzyme concentration $\left([S]_{0}>>[E]_{t}\right)$.

$$
\text { velocity }=k_{\text {cat }}[E]_{t} \frac{[S]}{[S]+K_{d}}
$$

This initial model was later updated by Briggs and Haldane in $1925^{16}$ and Morrison in 1969 to cover a comprehensive set of conditions. ${ }^{17}$ Over the past 100 years, these binary models and saturation behavior has provided the conceptual foundations for investigators studying enzymatic processes ranging from intracellular signaling to clinical pharmacokinetics. ${ }^{18,19}$

\section{Simplified Models of Ternary Enzyme Catalysis}

Ternary enzyme catalysis, on the other hand, has proven more difficult to quantitatively define to due to the combinatorial complexity of enzyme/target binding (Figure 1B). ${ }^{20}$ First, two binding events are 
necessary, as the bifunctional molecule must simultaneously bind the enzyme $\left(K_{E B}\right)$ and the target $\left(K_{B T}\right)$ to facilitate enzymatic modification of the target. Second, physical interactions between the enzyme and target must be described by an additional energetic parameter called cooperativity $(\alpha)$. This interaction can be either stabilizing $(\alpha>1)$ or destabilizing $(\alpha<1)$, depending on the complementarity of any enzymetarget interactions (Figure 1B). ${ }^{21}$ As a result, most attempts to mathematically model ternary complex kinetics have been based on numerical simulations or simplifying assumptions. For example, models of inter-cellular signaling have generally assumed that the bifunctional molecule (e.g. cytokine, hormone) is in excess of cell-surface receptor concentrations $([B]>[E],[T]) .^{22}$ Another common assumption is that the target is the limiting reagent $([E],[B]>>[T])$, which has been used to model the anticoagulated effects of heparin and sandwich immunoassays. ${ }^{4,23}$ Finally, the assumption that both the enzyme and target are present in very high concentrations has been used in recent models of scaffold proteins kinetics ([E],[T] $>[B]) .^{2}$ These simplified models provide insights in specific systems, but these assumptions limit the scope of each mathematical model to a subset of biological processes.

\section{Bell-Shaped Dose-response curves}

In addition to this mathematical complexity, titrations of bridging molecule (B) result in a non-intuitive "bell-shaped dose-response curve" (Figure S1-S2) that is distinct from saturation behavior of classical enzyme kinetics (Figure 1C-D). ${ }^{21}$ This is a major conceptual difference between binary and ternary systems, as many ternary systems can only reach a fraction of the theoretical maximum enzymatic flux $\left(k_{c a t}[E]_{t}\right)$. Critically, this means that ternary complex based drugs will have an intrinsic maximum effect dose $\left([B]_{t, \max }\right)$, and that at this dose the fraction of possible ternary complex formed may not saturate the enzyme. This inherent phenomenon of TEC can limit the efficacy ( $y$-axis magnitude) of bifunctional drugs (Figure 2A). This autoinhibitory behavior has been directly observed for several clinical therapies including: growth hormones, cytokines, monoclonal antibodies, heparin, and most recently PROTAC's and bispecific antibodies. ${ }^{24-28}$

\section{Ternary Complex Equilibrium Models}

In 2013, we published the first exact equilibrium model for ternary complex equilibria. ${ }^{21} \mathrm{~A}$ critical finding of this work was that the weakest binding affinity $\left(K_{\text {weak }}\right)$ is generally most important binding parameter for increasing the fraction of ternary complex that forms at equilibrium. More specifically, improving $K_{\text {weak }}$ can improve both the potency (x-axis) and efficacy (y-axis) properties of a drug's dose-response curve, whereas improvements in $K_{\text {strong }}$ only improves the potency of the drug (Figure S3). This prediction has been borne out experimentally from our work designing synthetic antibodies (Figure S4). ${ }^{21,29}$ In addition, optimizing $K_{\text {weak }}$ has been noted by several other investigators to be critical to improving other ternarycomplex therapies including: cytokines, heparin and antibody-based therapeutics. ${ }^{4,5,30-33}$

\section{Manuscript Summary}

Though an important advance, this "Hill equation" for ternary complex equilibria is not sufficient to describe the kinetics of ternary-complex therapeutics which redirect enzymatic activity. Here we extend these equilibrium models to derive general "Michaelis-Menten" equations (Figure 2A) for ternarycomplex mediated catalysis (Figure 2B-C). These equations provide a simple conceptual framework for understanding the timescales of ternary complex mediated catalysis and reconciles a diverse array of experimental literature on the kinetics of TEC-based therapeutics. 


\section{$\mathbf{V}_{\max }$ of Scaffolded Enzyme Catalysis}

\section{Results and Discussion}

The velocity of ternary-complex mediated enzymatic reactions is proportional to rate constant for the enzymatic process $\left(k_{c a t}\right)$ and the concentration of ternary complex in solution ([EBT]):

$$
\text { velocity }=k_{\text {cat }}[E B T]
$$

In addition, the maximum velocity of ternary-catalysis $\left(V_{\max }\right)$ should occur under conditions where maximum ternary complex forms $\left([E B T]_{\max }\right)$ :

$$
V_{\text {max }}=k_{c a t}[E B T]_{\max }
$$

As noted above, the theoretical upper limit on $V_{\max }$ for SEC is $k_{c a t}[E]_{t}$, but in many systems this limit is not reached at any concentration of B (Figure 1D).

\section{Pre-equilibrium Ternary Kinetic Models}

For ternary systems in pre-equilibrium conditions, the equilibration of the complex occurs faster than the transformation by the enzyme $\left(k_{\text {cat }}\right) .{ }^{20}$ This assumption is reasonable for many natural and most synthetic systems. ${ }^{20}$ Indeed, two recent kinetic analyses of PROTACs concluded that they were in a pre-equilibrium regime $^{34,35}$. Assuming pre-equilibrium, it has been previously shown that $[E B T]_{\max }$ can be calculated with

A. Integrated Michaelis-Menten Equation:

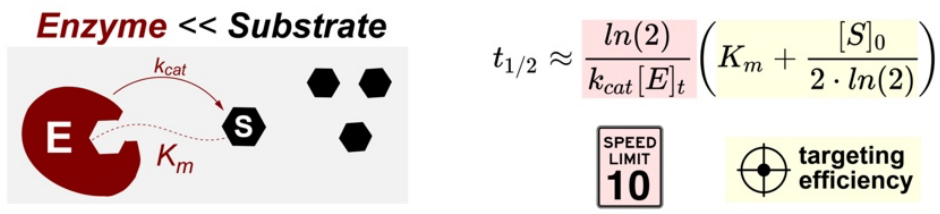

B. Quadrant Framework for Relative Conditions of Ternary Complex Maxima

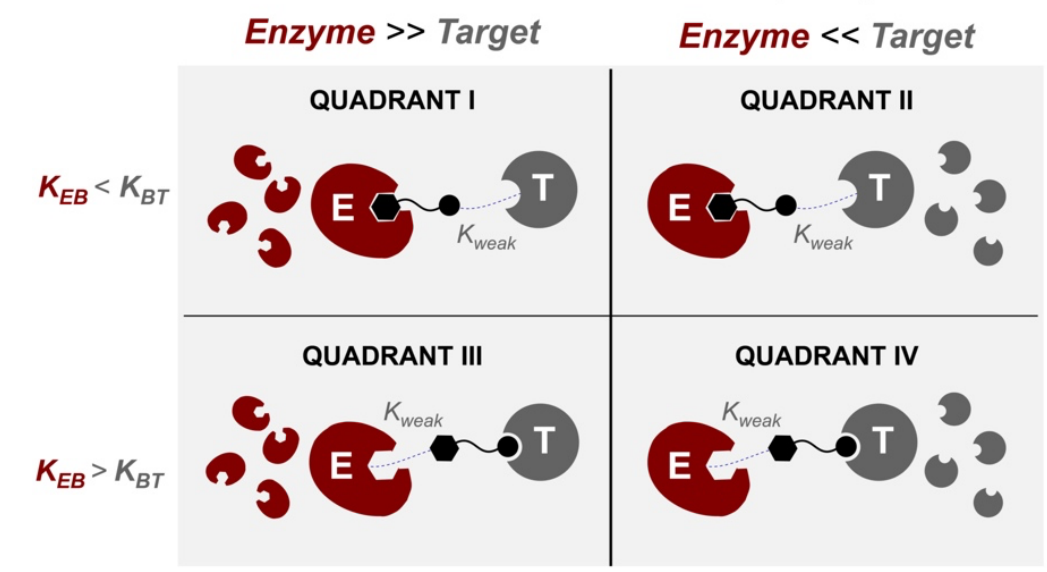

C. Integrated Ternary Vmax Equation:

$$
\begin{array}{c|c}
\text { Enzyme }>\text { Target } & \text { Enzyme }<<\text { Target } \\
t_{1 / 2} \approx \frac{\ln (2)}{k_{\text {cat }}[E]_{t}}\left(\frac{K_{\text {weak }}}{\alpha}+[E]_{t}\right) & t_{1 / 2} \approx \frac{\ln (2)}{k_{\text {cat }}[E]_{t}}\left(\frac{K_{\text {weak }}}{\alpha}+\frac{[T]_{0}}{2 \cdot \ln (2)}\right)
\end{array}
$$

Figure 2. Integrated kinetic models of $V_{\max }$ ternary-complex mediated catalysis. A. The integrated Michaelis-Menten equation can be understood as having two parts: an intrinsic "speed limit" determined by the concentration and intrinsic rate of the enzyme and a targeting efficiency term that describes the fraction of enzyme bound. B. The integrated form of the ternary Vmax equation has a very similar form with differences depending on whether or not $\mathrm{E}>>\mathrm{T}$ or $\mathrm{E}<<\mathrm{T}$ (Michaelis-Menten assumes $\mathrm{E}<<\mathrm{S}$ ).

$$
[E B T]_{\text {max }}=[L]_{t} \frac{[X]_{t}}{[X]_{t}+K_{\text {weak }} / \alpha}
$$

Where $[L]_{t}$ and $[X]_{t}$ represents the concentrations of limiting and excess terminal species $(E$ or $T)$ and $K_{\text {weak }}$ represents the weaker of the two binding affinities $\left(K_{E B}\right.$ or $\left.K_{B T}\right)$. Equation 4 may appear overly abstract, but has classical precedent in the Langmuir-Hill equation where the receptor is assumed to be limiting $[L]_{t}$ and the ligand is assumed to be in excess $\left([X]_{t}\right)^{36,37}$

$$
[L X]=[L]_{t} \frac{[X]_{t}}{[X]_{t}+K_{d}}
$$

At $[E B T]_{\max }$, the equilibrium can be conceptualized as a binary complex (either EB or BT) searching for the (weaker binding) third partner (Figure 2B). As detailed in the supporting information, at $[E B T]_{\max }$, the stronger binding side of $B$ will be predominately bound while the weaker binding will predominately be unbound. This physical picture of [EBT] $\max$ can be separated into a four-quadrant framework defining the 4 possible combinations of limiting reagents and weaker binding affinities (Figure 2B). Quadrants I \& III capture experimental conditions when the enzyme is in excess of the target, while quadrants II and IV capture conditions when the target in in excess of the enzyme (most analogous to Michaelis-Menten 
assumption). Overall, equation 4 can be conceptualized as a preformed dimer is searching for the third - weaker binding - partner scaled by the cooperativity in forming the complex $\left(K_{\text {weak }} / \alpha\right)$.

\section{Timescale of Ternary-complex mediated catalysis}

Replacing the $[E B T]_{\max }$ term in equation 4 we obtain a general equation for $V_{\max }$ for ternary complex catalysis:

$$
V_{\text {max }}=k_{\text {cat }}[L]_{t} \frac{[X]_{t}}{[X]_{t}+K_{\text {weak }} / \alpha}
$$

Equation 6 has a similar form to classical Michaelis-Menten kinetic equation (equation 1), but is more general as the enzyme can be either limiting $\left([\mathrm{L}]_{t}\right)$ or in excess $\left([\mathrm{X}]_{t}\right)$. While this differential equation can be useful for mathematical modeling, solving for the half-life of catalysis can provide a more intuitive way to understand the system. This approach was pioneered by Michaelis and Menten in their original 1913 publication (equation 7) but has gotten surprisingly little attention within the scientific literature (Figure 2A): ${ }^{15,38}$

$$
t_{1 / 2}=\frac{\ln (2)}{k_{\text {cat }}[E]_{t}} \times\left(\frac{K_{m}}{\alpha}+\frac{[S]_{0}}{2 \cdot \ln (2)}\right)
$$

Inspired by Michaelis-Menten's original paper, we integrated equation 6 with respect to time and the concentration of target $(\mathrm{T})$. As []$_{0}$ can be in excess $\left([\mathrm{X}]_{t}\right)$ or limiting $\left([\mathrm{L}]_{t}\right)$, it is necessary to integrate equation 6 for both conditions (Figure 2C), as is detailed in the supporting information(Figure S5-6). When target concentration is higher than the enzyme concentration $\left([\mathrm{T}]_{0}>>[E]_{t}\right)$, the half-life can be approximated by equation 8 (Figure S5, eq S69):

$$
t_{1 / 2}^{\min }=\frac{\ln (2)}{k_{\text {cat }}[E]_{t}} \times\left(\frac{K_{\text {weak }}}{\alpha}+\frac{[T]_{0}}{2 \cdot \ln (2)}\right)
$$

When the enzyme concentration is higher than the target concentration $\left([T]_{0}<<[E]_{t}\right)$, the half-life can be approximated by equation 9 (Figure S6, es S77):

$$
t_{1 / 2}^{\min }=\frac{\ln (2)}{k_{\text {cat }}[E]_{t}} \times\left(\frac{K_{\text {weak }}}{\alpha}+[E]_{t}\right)
$$

Strikingly, equations 7-9 all have a similar form. The left side represents a kinetic "speed limit" equal to the time-scale of catalysis when an enzyme is saturated. The right hand-side can be interpreted as the "targeting efficiency", or fraction of enzyme bound to target/substrate at any given time (Figure 2A). As detailed in the supporting information, both equation 8 and 9 can be simplified to the same form if one assumes pseudo-first order conditions (i.e., neither E or T are saturated) to yield equation 10:

$$
t_{1 / 2}^{\min } \approx \frac{\ln (2)}{k_{\text {cat }}[E]_{t}} \times\left(\frac{K_{\text {weak }}}{\alpha}\right)
$$

As discussed below, equation 10 provides a simple conceptual framework to reconcile a wide variety of literature on the timescales of ternary-complex mediated catalysis.

\section{Heparin-mediated deactivation of Thrombin}

One of the most well characterized ternary-complex therapeutics is heparin, which acts as an anticoagulant by forming a ternary complex with antithrombin (E) and thrombin $(T)$ to drive covalent modification and deactivation of Thrombin (Figure 3A). The high affinity heparin parameters are $K_{E T}=$ $10 \mathrm{nM}, K_{B T}=100 \mathrm{nM}, \alpha=1$, and $k_{c a t}=5 \mathrm{~s}^{-1}$ (Figure 3B), which results in faster catalysis than lower affinity variants where $K_{E T}=10,000 \mathrm{nM}, K_{B T}=1,000 \mathrm{nM}$ and $k_{c a t}=5 \mathrm{~s}^{-1}$ (Figure 3C). Unlike many clinical TEC therapeutics, numerous examples of bell-shaped dose-velocity curves have been measured for this system. ${ }^{3,4}$ providing a unique opportunity to directly assess the utility of our kinetic models, as $V_{\max }$ can be directly inspected from these curves (Figure 3B-C). 


\section{A. Heparin Kinetics in vitro: pseudo-first order pre-equilibrum}

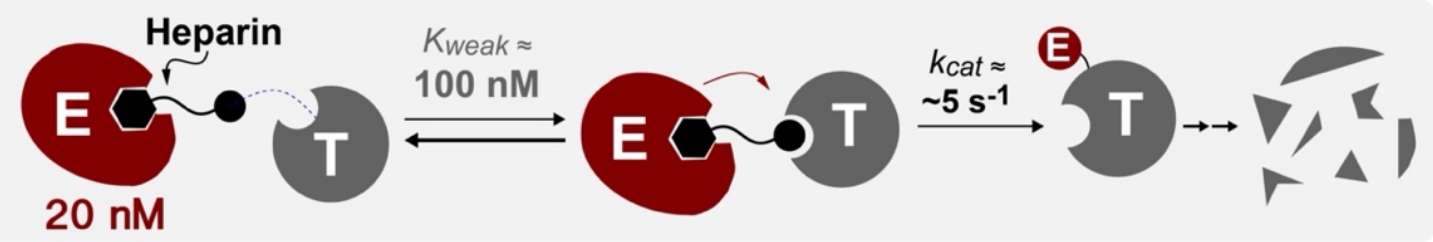

$$
t_{1 / 2} \approx \frac{\ln (2)}{k_{c a t}[E]_{t}}\left(\frac{K_{w e a k}}{\alpha}\right) \approx 1 \mathrm{~s}
$$

B. High Affinity Heparin (100nM)

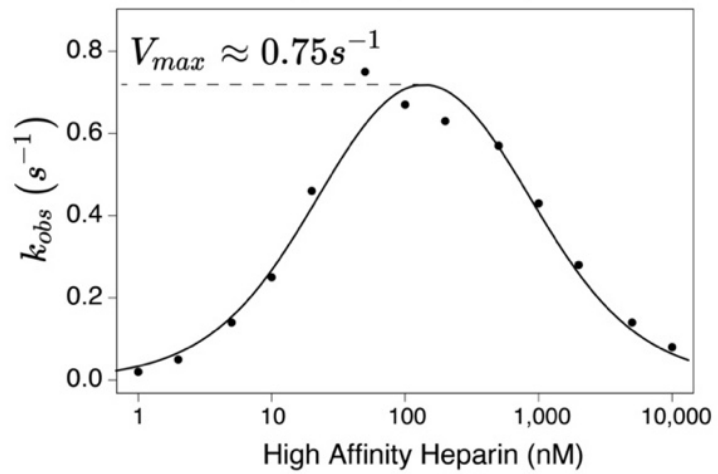

C. Low Affinity Heparin $(\mathbf{1 0} \mu \mathrm{M})$

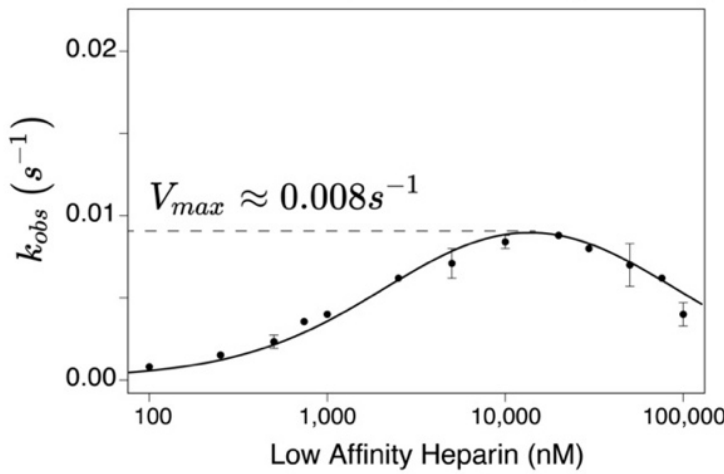

D. PROTACs Kinetics: pseudo-first order pre-equilibrum

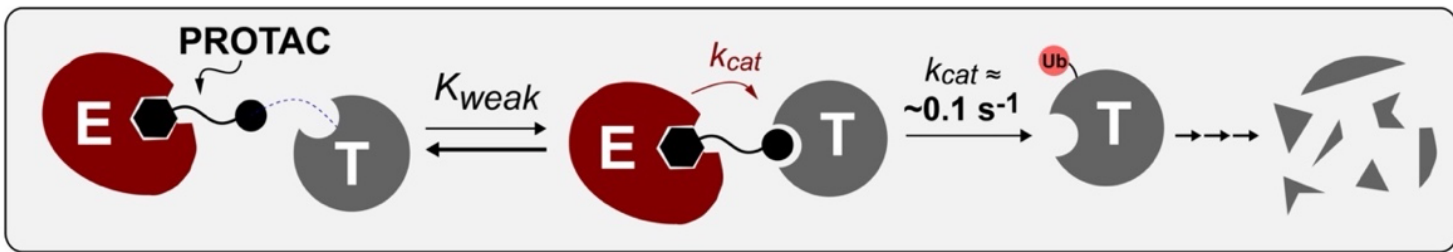

$$
t_{1 / 2} \approx \frac{\ln (2)}{k_{c a t}[E]_{t}}\left(\frac{K_{\text {weak }}}{\alpha}\right) \approx 180 \min
$$

E. $[E] t$ variability

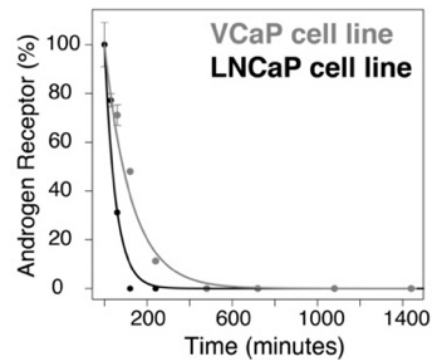

F. Dose variability

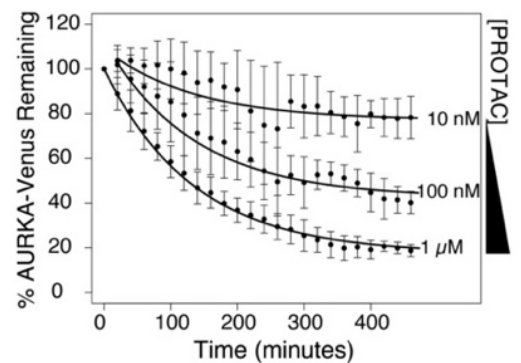

G. Kweak/a variability

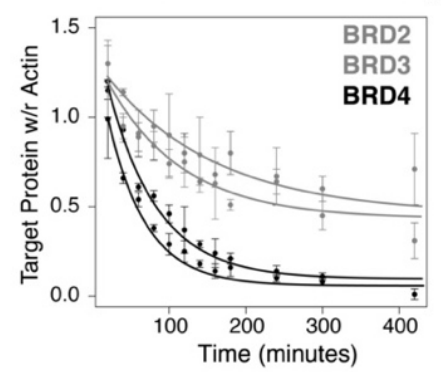

Figure 3. Reconciliation of Diverse Scientific Literature on Ternary Complex Mediated catalysis. A. Mechanism of High affinity Heparin's anti-Thrombin activity B. High affinity heparin is expected to have a Vmax time-scale of approximately $1 \mathrm{~s}$ when the Antithrombin(E) concentration is $20 \mathrm{nM}^{4}{ }^{4}$ C. Low affinity heparin is expected to have a time-scale of approximately $100 \mathrm{~s}$ when the Antithrombin concentration is $20 \mathrm{nM} .{ }^{4}$ D. Mechanism of PROTAC-mediated proteolytic degradation of target proteins E. PROTAC activity timescales depend on cellular context (i.e. protein expression). ${ }^{39} \mathbf{F}$. PROTAC activity timescales depend on PROTAC dose ${ }^{40}$ G. PROTAC activity timescales depend on target affinity ${ }^{34}$ 
Encouragingly, we find a striking concordance between the predictions of equation 10 and dosevelocity data presented in Figure 3B-C. ${ }^{4}$ For example, parameterizing equation 10 with known values for heparin, we would expect a time-scale for deactivation of thrombin of approximately 1 second:

$$
t_{1 / 2}^{\min } \approx \frac{\ln (2)}{k_{\text {cat }}[E]_{t}} \times\left(\frac{K_{\text {weak }}}{\alpha}\right) \approx \frac{\ln (2)}{5 s^{-1} 20 n M} \times\left(\frac{100 n M}{1}\right) \approx 1 s
$$

Inspection of Figure 3B reveals that this almost exactly corresponds to the effective timescale observed at $V_{\max }\left(k_{\max }^{\text {obs }} \approx 1 / t_{1 / 2}^{\min }\right)$. In addition, for Low affinity Heparin the only parameter in equation 11 which would change would be $K_{\text {weak }}$ which changes $100 x$ from $100 \mathrm{nM}$ to $10,000 \mathrm{nM}$. This should correspond to a $100 x$ reduction in $k_{o b s}$ which is exactly what is observed in Figure $\mathbf{3 C}$. Interestingly, this correlation between $K_{\text {weak }}$ and the relative rates of reaction was directly noted by the authors of this study as well as other kinetics studies on the signaling of interferons. ${ }^{4,32}$ Overall, our new theory provides a physical rationale for the previously empirical observation that $K_{\text {weak }}$ best correlates with catalytic time-scales.

\section{Timescale of synthetic molecule mediated ternary-catalysis}

As mentioned above, the recent successes of PROTAC's and bispecific antibodies has driven an expansion in interest in synthetic molecules which engineer new modes of ternary complex catalysis. Unlike natural products, these molecules have a wide range of binding affinities and target concentrations, and so we have sought to define the normal range of affinities and concentrations by collected data from several large

databases (Figure 4). For example, the ChEMBL database has begun to define the typical range of drug-target $K_{d}$ 's by curating $>60,000$ medicinal chemistry studies (Figure 4a), demonstrating that normal affinities tend to be centered around 100 $\mathrm{nM}^{41}$ In addition, recent quantitative proteomics work has identified the average intracellular protein concentration as around $\sim 10 \mathrm{nM}$ (Figure 4a). ${ }^{42,43}$ In addition to this thermodynamic data, several datasets on kinetic parameters exist as well. For example, the BRENDA database has catalogued over 33,000 enzymatic rate constants $\left(k_{\text {cat }}\right)$, eastablinging mean enzymatic turnover emerges as around 10x per second (Figure 4b). ${ }^{44}$

By combining our preequilibrium model with literature values and typical physiological values, we can estimate the halflife of a "normal" PROTAC target.

a. Normal equilibrium values: $K d$ and protein concentration
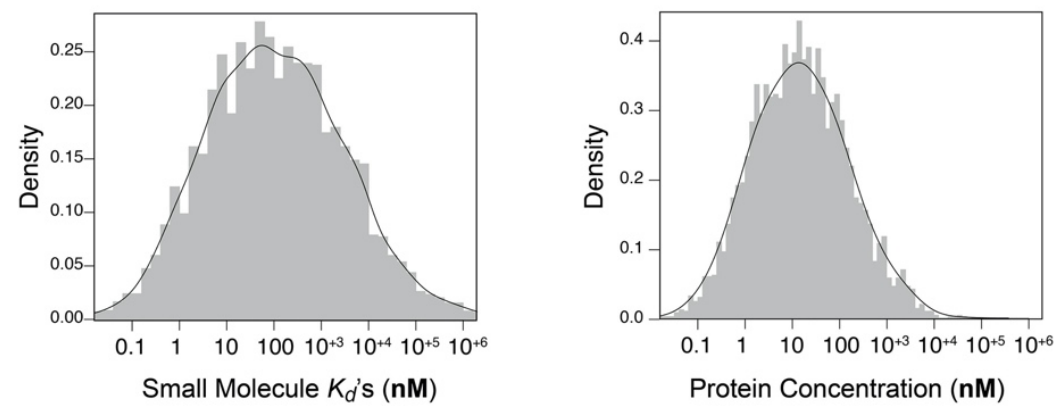

b. Normal kinetic values: $k_{c a t}$ and protein half-life
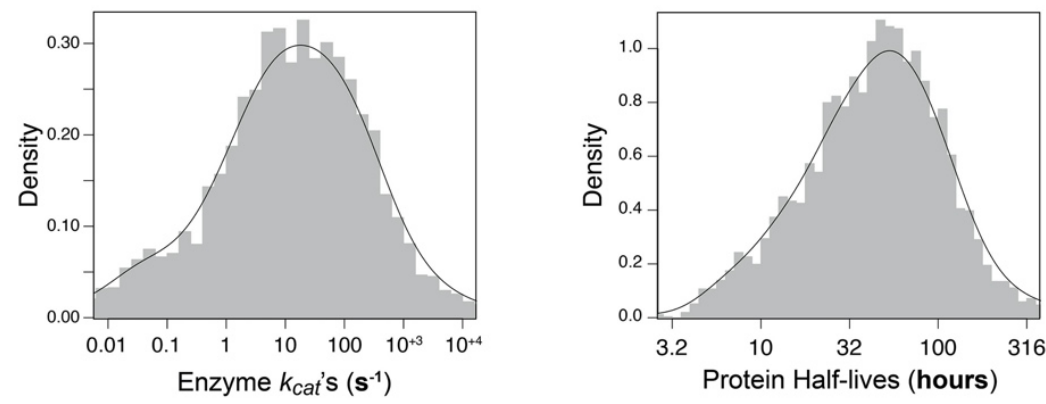

Figure 4. Reasonable ranges for parameter values can be estimated based on databases. a. Normal range of dissociation constants and protein concentrations compiled from medicinal chemistry studies and quantitative proteomics. b. Normal range of enzymatic rate constants and protein half-lives compiled from the biochemistry literature and quantitative proteomic study.

Typical saturated ubiquitination kinetics for E3 ligases occurs with a rate constant $\left(k_{\text {cat }}\right)$ of approximately $0.1 \mathrm{~s}^{-1} .{ }^{45}$ Conservatively assuming the E3 ubiquitin ligase is expressed at $1 \mathrm{nM}$ and the PROTAC $K_{\text {weak }}$ $\approx 1 \mu \mathrm{M}$, we would expect a ubiquitination time-scale on the order of approximately 3 hours or 180 minutes: 


$$
t_{1 / 2}^{\min } \approx \frac{\ln (2)}{k_{\text {cat }}[E]_{t}} \times\left(\frac{K_{\text {weak }}}{\alpha}\right) \approx \frac{\ln (2)}{0.1 \mathrm{~s}^{-1} 1 \mathrm{nM}} \times\left(\frac{1,000 \mathrm{nM}}{1}\right) \approx 10,000 \mathrm{~s} \approx 180 \mathrm{~min}
$$

This half-life is consistent with recent kinetic studies on PROTAC kinetics (Figure 3 E-G). ${ }^{34,35,39,40}$ In addition, these three studies demonstrate sensitivity of timescales to enzyme concentration, bifunctional molecule dose and target affinities (Figure 3E-G), providing further support for the validity of our mathematical model. Critically, this $\sim 3$ hour time-scale is $16 \mathrm{x}$ faster than the than the typical rate of protein turnover $(\sim 48 \text { hours, Figure } 4 \mathbf{b})^{42}$ which explains why PROTAC's can exhibit efficacy even with modest binding affinities $\left(K_{d} \sim 1 \mu \mathrm{M}\right)$.

\section{Timescales of antibody-based drugs}

While the above models are only directly relevant to ternary enzyme catalysis, they still reconcile a wide variety of literature on the molecular determinants of antibody-based drug efficacy (Figure 5). For example, antibody-dependent cellular cytotoxicity (ADCC) is generally dependent on the formation of a ternary complex between a bifunctional antibody (B) and FCReceptors on an effector cell (E) and antigens on a target cell $(\mathrm{T})$. Upon formation of a ternary complex between the effector and target cell, cell-killing has been shown to be initiated a time-scale of approximately 1 hour (Figure 5A). ${ }^{47}$ For monoclonal antibodies, Fc-receptor affinity can be generally taken as $K_{\text {weak }}$ as it has been shown that $\mathrm{FcR} / \mathrm{lgG}$ affinity is approximately $\sim 1 \mu \mathrm{M}$ while affinity maturation has typically results in IgG/Target affinities $\sim 1 \mathrm{nM} .^{48,49}$ This would indicate that - at $V_{\max }$ - the target cell should be saturated with antibody (i.e. opsonized) while effector cells should remain largely unbound and thus free to engage multiple types of pathogenic cells. This provides a functional explanation for the relatively low antibody/FcR affinity as it prevents effector cells from being saturated by a wide variety of IgG present in serum. As predicted by our model, modification of antibodies that increase the FcR affinity ( $\left.K_{\text {weak }}\right)$ have been shown to significantly increase ADCC across multiple studies. ${ }^{5,33,50}$

A critical feature that distinguishes ADCC from the TEC model presented above is multi-

A. Antibody Dependent Cellular Cytotoxicity Model:
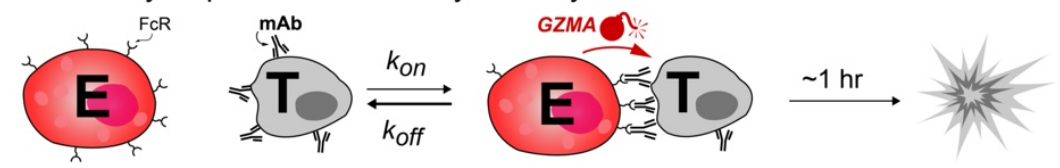

B. Receptor Dependence of Cytotoxicity for 20nM bispecific Antibody CEA-TCB

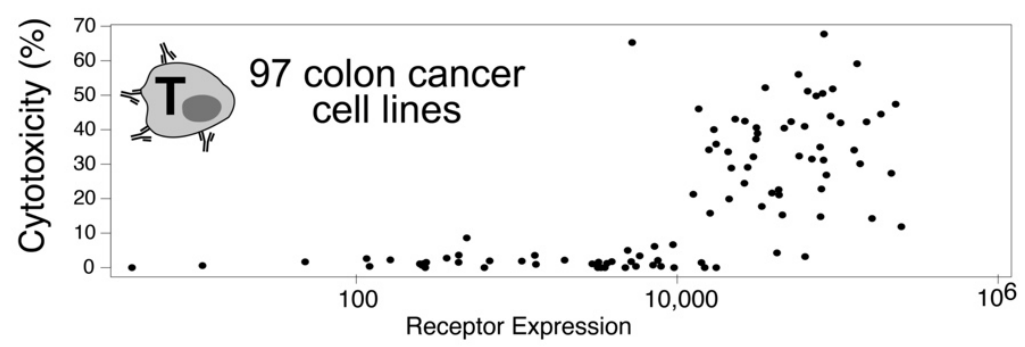

C. Maximum Rates of Cytotoxicity (limit as $\alpha \rightarrow \infty$ )

$\mathrm{E}: \mathrm{T} \gg 1$

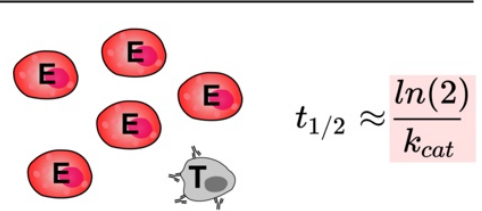

D. Cytotoxicity Kinetics (E:T $=50: 1)$

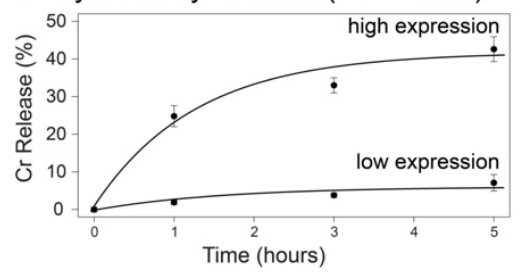

Figure 5. Reconciliation of scientific literature on the molecular determinants of ATCC of monoclonal and bispecific antibodies. A. Expected mechanism of ADCC for monoclonal antibodies B. Antigen expression dependence of ADCC for a bispecific antibody across 97 cell lines C. Maximum rates of cytotoxicity estimated by taking the limit of positive cooperativity $\mathbf{D}$. At high $\mathrm{E}: \mathrm{T}$ ratios the time-scale of cytotoxicity is approximately equal to 1 hour $^{46}$ E. At high E:T ratios mAb's exhibit a bell-shaped dose response $^{25}$

valent antibody binding across the effector/target contact site (Figure 5A). At sufficiently high receptor densities, this "zippering" can significantly increase the time-scale of cellular adhesion. ${ }^{51,52}$ In fact, one 
recent study demonstrated that ADCC is not possible less than 10,000 receptors/cell (Figure $\mathbf{5 B}$ ). ${ }^{53,54}$ Within the context of this model, these additional E-T interactions represent a dimensionless stabilization energy and can be conceptually interpreted as an additional stabilizing cooperativity. ${ }^{52}$ While others have calculated this stabilizing/destabilizing energy directly, ${ }^{52}$ here we can obtain a rough time-scale for ADCC for target cells with $>10,000$ antigens by taking the limit of equations 8 and 9 as cooperativity increases to obtain expressions for ADCC time-scales when the effector to target ratio (E:T) is greater than or less than 1 (Figure $\mathbf{5 C}$ ). When the E:T >> 1, the target will be modified on approximately the same time-scale as $k_{\text {cat }}(\sim 1$ hour $)$ :

$$
t_{1 / 2}^{\min }=\lim _{\alpha \rightarrow \infty}\left(\frac{\ln (2)}{k_{\text {cat }}[E]_{t}} \times\left(\frac{K_{\text {weak }}}{\alpha}+[E]_{t}\right)\right)=\frac{\ln (2)}{k_{\text {cat }}}
$$

On the other hand, when E:T $<1$, the time-scale of cytotoxicity will be scaled up by the target/effector ratio:

$$
t_{1 / 2}^{\min }=\lim _{\alpha \rightarrow \infty}\left(\frac{\ln (2)}{k_{\text {cat }}[E]_{t}} \times\left(\frac{K_{\text {weak }}}{\alpha}+\frac{[T]_{0}}{2 \cdot \ln (2)}\right)\right)=\frac{1}{k_{\text {cat }}[E]_{t}} \times\left(\frac{[T]_{0}}{2}\right)
$$

While an over simplification, these equations provide a kinetic rationale for the E:T variability of ADCC in the context of in vitro assays. In addition, time-scale of approximately 1 hour are consistent with multiple studies that have both monitored cytotoxicity over time and employed large E:T ratio of approximately 50:1 (Figure 5E-F). ${ }^{25,46,55}$

\section{Discussion}

Over 100 years ago Langmuir and Hill laid the foundation for modern pharmacology with their derivation of the equilibrium binding of a ligand and its receptor (equation 5). ${ }^{36,37,56}$ The key conceptual insight from their work was that titrations of ligand exhibit S-shaped dose-response curves as a result of saturation of their target receptor (Figure S1). Shortly after, Michaelis and Menten extended this "saturation" conceptual framework to enzyme kinetics, laying the conceptual foundation for quantitative biochemistry and clinical pharmacokinetics (equation 1)..$^{14,15}$ Since then, an increasing number of biological processes and clinical therapies have been shown to operate via indirect interactions between an enzyme and its target via an intermediate bridging species (B). These systems include: most cytokines, several MAP-kinase cascades, and several immune-responses. ${ }^{3-8}$ Unfortunately, the mathematical complexity of these systems has prevented the development of a single unified model (analogous to Michaelis-Menten) to quantitatively define these processes.

Here, by focusing on $V_{\max }$ and adapting an underappreciated insight of Michaelis and Menten (integration of the velocity equation), ${ }^{38}$ we have derived a general model that reconciles a wide variety of literature on ternary-complex mediated enzyme catalysis (TEC). This model identified the enzymatic rate constant $\left(k_{c a t}\right)$, the enzyme concentration $\left([E]_{t}\right)$, and the weakest binding affinity $\left(K_{\text {weak }}\right)$ as the most important parameters to engineer ternary-complex mediated catalytic timescales.

Today, the need for such models is particularly urgent given the increasing interest in the development of bifunctional drugs that redirect or "rewire" various enzymatic and immune functions to treat disease. The analysis presented here predicts that there are several ways to increase the rate of TEC for therapeutics. First, a bifunctional drug must be dosed in the high velocity range (near $V_{\max }$ ). Our previous work discusses in detail how to predict the concentration of B that will produce the most ternary complex, and thus approach $V_{\max }$ for the system. ${ }^{21}$ Second, improving the $K_{\text {weak }}$ of the bifunctional drug should increase the SEC rate by increasing the theoretical fraction of ternary complex that forms, allowing the system to approach the theoretical kinetic ceiling of $k_{\text {cat }}[\mathrm{E}]_{t}$ (Figure 1D). Third, choosing a more abundant or efficient enzyme can improve TEC rate by raising that kinetic ceiling itself (Figure 1D). As noted by several recent PROTAC reviews, this provides an opportunity for tissue selectivity. ${ }^{11,28}$ For 
example, if the E3 ligase targeted by a PROTAC is at much higher concentration in a tissue, then the target will be degraded much more efficiently in that tissue than those with lower enzyme concentration. ${ }^{11}$ Similarly, targeting more abundant immune effector cell types (i.e. E:T ratio) has been shown to increase the rate of ADCC. ${ }^{55}$

Given the combinatorial complexity of possible experimental conditions (Figure 2B) we have designed a webtool to automate the time-scale analysis described throughout this paper: https://douglasslab.com/btmax kinetics/. This tool is designed to streamline the logical flow for noncomputational investigators by enabling (1) graphical entry of experimental conditions (2) automatic assignment of appropriate half-life equation to those conditions. To limit the error in the half-life estimate in the online application, we have replaced $K_{\text {weak }}$ with a more precise estimate of ternary affinity $\left(\sqrt{K_{E B}}+\sqrt{K_{B T}}\right)^{2}$ as detailed in our original equilibrium modeling work. ${ }^{21}$ For example, equation 10 would be more precisely approximated by equation $15 .^{21}$

$$
t_{1 / 2}=\frac{\ln (2)}{k_{c a t}[E]_{t}} \times \frac{\left(\sqrt{K_{E B}}+\sqrt{K_{B T}}\right)^{2}}{\alpha}
$$

For the online applications, we chose to place the system concentrations and $K_{d} S$ on the same logarithmic scales and in the same units $(\mathrm{nM})$ to emphasize how their relative values affect the ternary system(Figure S7). We have confined these values to reasonable ranges from the literature values (Figure 4). Overall, this tool combines first principles-based kinetics models, with "big data" knowledge of affinities, concentrations and rate constants to enable non-computational investigators to apply the conceptual framework described here.

This mathematical framework is based on similar underlying assumptions as Michealis-Menten kinetics and will likely have similar scope (and limitations). Encouragingly, examination of the literature on TEC has demonstrated a surprising amount of correspondence with these simple equations and direct kinetic measurements (Figure 3). More generally, we hope that this work will bring more attention to the power Michaelis and Menten's initial insight to integrate their velocity equations to characterize the timescales of kinetic processes (Figure 2A). In fact, this integrated approach is already employed by clinical pharmacists to conceptualize the [largely] enzymatic clearance $(\mathrm{CL})$ of drugs from the body's volume of distribution $(\mathrm{V}):^{18,19}$

$$
t_{1 / 2}=\frac{\ln (2)}{C L} \times V
$$

We believe, that a wider appreciation of the integrated Michaelis-Menten equation (Figure 2A) will put the tools of enzyme kinetics into the hands of a much wider range of investigators than the differential model alone (equation 1). ${ }^{38}$

Overall, this work represents a reconceptualization of pharmacodynamics from a static (LangmuirHill equation) to a dynamic process based the same concepts that underly pharmacokinetics. While most drugs act as inhibitors, an increasing number of biological and small molecule therapies act as agonists, inducing a therapeutic phenotype with a characteristic time-scale. While our work focuses on ternary-complex based agonists, we believe it is part of a general trend to reconceptualize drugmechanism from a static to kinetic process which requires new conceptual frameworks that describe drug-mechanism as more than simple binding to a target. 



\section{References}

1 Good, M. C., Zalatan, J. G. \& Lim, W. A. Scaffold Proteins: Hubs for Controlling the Flow of Cellular Information. Science 332, 680-686, doi:10.1126/science.1198701 (2011).

2 Levchenko, A., Bruck, J. \& Sternberg, P. W. Scaffold proteins may biphasically affect the levels of mitogen-activated protein kinase signaling and reduce its threshold properties. $P$ Natl Acad Sci USA 97, 5818-5823, doi:DOI 10.1073/pnas.97.11.5818 (2000).

3 Griffith, M. J. Kinetics of the Heparin-Enhanced Antithrombin-lii Thrombin Reaction - Evidence for a Template Model for the Mechanism of Action of Heparin. J Biol Chem 257, 7360-7365 (1982).

4 Streusand, V. J., Bjork, I., Gettins, P. G. W., Petitou, M. \& Olson, S. T. Mechanism of Acceleration of Antithrombin-Proteinase Reactions by Low-Affinity Heparin. J Biol Chem 270, 9043-9051, doi:DOI 10.1074/jbc.270.16.9043 (1995).

5 Carter, P. J. \& Lazar, G. A. Next generation antibody drugs: pursuit of the 'high-hanging fruit'. Nat Rev Drug Discov 17, 197-223, doi:10.1038/nrd.2017.227 (2018).

6 Labrijn, A. F., Janmaat, M. L., Reichert, J. M. \& Parren, P. Bispecific antibodies: a mechanistic review of the pipeline. Nat Rev Drug Discov 18, 585-608, doi:10.1038/s41573-019-0028-1 (2019).

7 Devos, A. M., Ultsch, M. \& Kossiakoff, A. A. Human Growth-Hormone and Extracellular Domain of Its Receptor - Crystal-Structure of the Complex. Science 255, 306-312, doi:DOI 10.1126/science.1549776 (1992).

8 Ilondo, M. M. et al. Receptor Dimerization Determines the Effects of Growth-Hormone in Primary Rat Adipocytes and Cultured Human Im-9 Lymphocytes. Endocrinology 134, 2397-2403, doi:DOI 10.1210/en.134.6.2397 (1994).

9 Schreiber, S. L. The Rise of Molecular Glues. Cell 184, 3-9, doi:10.1016/j.cell.2020.12.020 (2021).

10 Gao, H., Sun, X. \& Rao, Y. PROTAC Technology: Opportunities and Challenges. ACS Med Chem Lett 11, 237-240, doi:10.1021/acsmedchemlett.9b00597 (2020).

11 Samarasinghe, K. T. G. \& Crews, C. M. Targeted protein degradation: a promise for undruggable proteins. Cell Chem Biol, doi:10.1016/j.chembiol.2021.04.011 (2021).

12 Carter, P. J. \& Lazar, G. A. Next generation antibody drugs: pursuit of the 'high-hanging fruit'. Nature Reviews Drug Discovery 17, 197-223, doi:10.1038/nrd.2017.227 (2018).

13 Mullard, A. Targeted protein degraders crowd into the clinic. Nature Reviews Drug Discovery 20, 247-250 (2021).

14 Michaelis, L., Menten, M. L., Johnson, K. A. \& Goody, R. S. The original Michaelis constant: translation of the 1913 Michaelis-Menten paper. Biochemistry 50, 8264-8269, doi:10.1021/bi201284u (2011).

15 Michaelis, V. L. \& Menten, M. M. L. Die kinetik der invertinwirkung (The Kinetics of Invertase Action). Biochemische Zeitschrift 49, 333-369 (1913).

16 Briggs, G. E. \& Haldane, J. B. S. A note on the kinetics of enzyme action. Biochem J 19, 338-339, doi:DOI 10.1042/bj0190338 (1925).

17 Morrison, J. F. Kinetics of Reversible Inhibition of Enzyme-Catalysed Reactions by Tight-Binding Inhibitors. Biochim Biophys Acta 185, 269-\&, doi:Doi 10.1016/0005-2744(69)90420-3 (1969).

18 Wagner, J. G. Properties of the Michaelis-Menten equation and its integrated form which are useful in pharmacokinetics. J Pharmacokinet Biopharm 1, 103-121, doi:10.1007/BF01059625 (1973). 
19 Rowland, M. \& Tozer, T. N. Clinical pharmacokinetics : concepts and applications. 3rd edn, (Williams \& Wilkins, 1995).

20 Segel, I. H. Enzyme kinetics : behavior and analysis of rapid equilibrium and steady state enzyme systems. (Wiley, 1975).

21 Douglass, E. F., Miller, C. J., Sparer, G., Shapiro, H. \& Spiegel, D. A. A Comprehensive Mathematical Model for Three-Body Binding Equilibria. J Am Chem Soc 135, 6092-6099, doi:10.1021/ja311795d (2013).

22 Perelson, A. S. Receptor Clustering on a Cell-Surface .2. Theory of Receptor Cross-Linking by Ligands Bearing 2 Chemically Distinct Functional-Groups. Math Biosci 49, 87-110, doi:Doi 10.1016/0025-5564(80)90112-1 (1980).

23 Rodbard, D., Feldman, Y., Jaffe, M. L. \& Miles, L. E. M. Kinetics of 2-Site Immunoradiometric (Sandwich) Assays .2. Studies on Nature of High-Dose Hook Effect. Immunochemistry 15, 77-82, doi:Doi 10.1016/0161-5890(78)90046-9 (1978).

24 Fuh, G. et al. Rational Design of Potent Antagonists to the Human Growth-Hormone Receptor. Science 256, 1677-1680, doi:DOI 10.1126/science.256.5064.1677 (1992).

25 Mulgrew, K. et al. Direct targeting of alpha(v)beta(3) integrin on tumor cells with a monoclonal antibody, Abegrin (TM). Mol Cancer Ther 5, 3122-3129, doi:10.1158/1535-7163.Mct-06-0356 (2006).

26 Taborda, C. P., Rivera, J., Zaragoza, O. \& Casadevall, A. More is not necessarily better: Prozonelike effects in passive immunization with IgG. J Immunol 170, 3621-3630, doi:DOI 10.4049/jimmunol.170.7.3621 (2003).

27 Betts, A. \& van der Graaf, P. H. Mechanistic Quantitative Pharmacology Strategies for the Early Clinical Development of Bispecific Antibodies in Oncology. Clin Pharmacol Ther 108, 528-541, doi:10.1002/cpt.1961 (2020).

28 Pettersson, M. \& Crews, C. M. PROteolysis TArgeting Chimeras (PROTACs) - Past, present and future. Drug Discov Today Technol 31, 15-27, doi:10.1016/j.ddtec.2019.01.002 (2019).

29 McEnaney, P. J. et al. Chemically Synthesized Molecules with the Targeting and Effector Functions of Antibodies. J Am Chem Soc 136, 18034-18043, doi:10.1021/ja509513c (2014).

30 Junttila, I. S. et al. Redirecting cell-type specific cytokine responses with engineered interleukin4 superkines. Nat Chem Biol 8, 990-998, doi:10.1038/Nchembio.1096 (2012).

31 Jaks, E., Gavutis, M., Uze, G., Martal, J. \& Piehler, J. Differential receptor subunit affinities of Type I interferons govern differential signal activation. J Mol Biol 366, 525-539, doi:10.1016/j.jmb.2006.11.053 (2007).

32 Kalie, E., Jaitin, D. A., Podoplelova, Y., Piehler, J. \& Schreiber, G. The Stability of the Ternary Interferon-Receptor Complex Rather than the Affinity to the Individual Subunits Dictates Differential Biological Activities. J Biol Chem 283, 32925-32936, doi:10.1074/jbc.M806019200 (2008).

33 Desjarlais, J. R. \& Lazar, G. A. Modulation of antibody effector function. Exp Cell Res 317, 12781285, doi:10.1016/j.yexcr.2011.03.018 (2011).

34 Roy, M. J. et al. SPR-Measured Dissociation Kinetics of PROTAC Ternary Complexes Influence Target Degradation Rate. Acs Chem Biol 14, 361-368, doi:10.1021/acschembio.9b00092 (2019).

35 Riching, K. M. et al. Quantitative Live-Cell Kinetic Degradation and Mechanistic Profiling of PROTAC Mode of Action. Acs Chem Biol 13, 2758-2770, doi:10.1021/acschembio.8b00692 (2018).

36 Langmuir, I. The constitution and fundamental properties of solids and liquids Part I Solids. J Am Chem Soc 38, 2221-2295, doi:DOI 10.1021/ja02268a002 (1916). 
37 Hill, A. V. The possible effects of the aggregation of the molecules of haemoglobin on its dissociation curves. Proceedings of the Physiological Society 40, 4-7 (1910).

38 Golicnik, M. The integrated Michaelis-Menten rate equation: deja vu or vu jade? J Enzyme Inhib Med Chem 28, 879-893, doi:10.3109/14756366.2012.688039 (2013).

39 Salami, J. et al. Androgen receptor degradation by the proteolysis-targeting chimera ARCC-4 outperforms enzalutamide in cellular models of prostate cancer drug resistance. Commun Biol 1, doi:ARTN 100

10.1038/s42003-018-0105-8 (2018).

40 Wang, R. et al. Selective targeting of non-centrosomal AURKA functions through use of a targeted protein degradation tool. Commun Biol 4, doi:ARTN 640

10.1038/s42003-021-02158-2 (2021).

41 Gaulton, A. et al. ChEMBL: a large-scale bioactivity database for drug discovery. Nucleic Acids Res 40, D1100-D1107, doi:10.1093/nar/gkr777 (2012).

42 Schwanhausser, B. et al. Global quantification of mammalian gene expression control. Nature 473, 337-342, doi:10.1038/nature10098 (2011).

43 Milo, R. \& Phillips, R. Cell biology by the numbers. (Garland Science, Taylor \& Francis Group, 2016).

44 Bar-Even, A. et al. The moderately efficient enzyme: evolutionary and physicochemical trends shaping enzyme parameters. Biochemistry 50, 4402-4410, doi:10.1021/bi2002289 (2011).

45 Pierce, N. W., Kleiger, G., Shan, S. O. \& Deshaies, R. J. Detection of sequential polyubiquitylation on a millisecond timescale. Nature 462, 615-U685, doi:10.1038/nature08595 (2009).

46 Velders, M. P. et al. The impact of antigen density and antibody affinity on antibody-dependent cellular cytotoxicity: relevance for immunotherapy of carcinomas. Brit J Cancer 78, 478-483, doi:DOI 10.1038/bjc.1998.518 (1998).

47 Murphy, K., Travers, P., Walport, M. \& Janeway, C. Janeway's immunobiology. 7th edn, (Garland Science, 2008).

48 Bruhns, P. et al. Specificity and affinity of human Fc gamma receptors and their polymorphic variants for human IgG subclasses. Blood 113, 3716-3725, doi:10.1182/blood-2008-09-179754 (2009).

49 Foote, J. \& Eisen, H. N. Kinetic and Affinity Limits on Antibodies Produced during ImmuneResponses. P Natl Acad Sci USA 92, 1254-1256, doi:DOI 10.1073/pnas.92.5.1254 (1995).

50 Romain, G. et al. Antibody Fc engineering improves frequency and promotes kinetic boosting of serial killing mediated by NK cells. Blood 124, 3241-3249, doi:10.1182/blood-2014-04-569061 (2014).

51 Lauffenburger, D. A. \& Linderman, J. J. Receptors : models for binding, trafficking, and signaling. (Oxford University Press, 1993).

52 Bell, G. I., Dembo, M. \& Bongrand, P. Cell-Adhesion - Competition between Nonspecific Repulsion and Specific Bonding. Biophys J 45, 1051-1064, doi:Doi 10.1016/S0006-

3495(84)84252-6 (1984).

53 Bacac, M. et al. A Novel Carcinoembryonic Antigen T-Cell Bispecific Antibody (CEA TCB) for the Treatment of Solid Tumors. Clin Cancer Res 22, 3286-3297, doi:10.1158/1078-0432.Ccr-15-1696 (2016).

54 Van de Vyver, A. J. et al. Predicting Tumor Killing and T-Cell Activation by T-Cell Bispecific Antibodies as a Function of Target Expression: Combining In Vitro Experiments with Systems Modeling. Mol Cancer Ther 20, 357-366, doi:10.1158/1535-7163.Mct-20-0269 (2021). 
55 Huang, G. L. et al. A multivariate, quantitative assay that disentangles key kinetic parameters of primary human T cell function in vitro. Plos One 15, doi:ARTN e0241421

10.1371/journal.pone.0241421 (2020).

56 Kenakin, T. P. A pharmacology primer : theory, applications, and methods. 3rd edn, (Academic Press/Elsevier, 2009). 